\title{
Diagnosis and treatment of a rare tumor-bladder paraganglioma
}

\author{
HANG LI $^{1,2}$, JIAODI XIE $^{1}$, ZEBO CHEN $^{1}$, SHANGQI YANG $^{1}$ and YONGQING LAI ${ }^{1,2}$ \\ ${ }^{1}$ Department of Urology, Institute of Urology, Peking University Shenzhen Hospital, \\ Shenzhen PKU-HKUST (Peking University, Hong Kong University of Science and Technology) Medical Center; \\ ${ }^{2}$ The Guangdong and Shenzhen Key Laboratory of Male Reproductive Medicine and Genetics, \\ Peking University Shenzhen Hospital, Shenzhen, Guangdong 518036, P.R. China
}

Received November 18, 2019; Accepted July 17, 2020

DOI: $10.3892 / \mathrm{mco} .2020 .2110$

\begin{abstract}
Bladder paragangliomas are very rare tumors that are often misdiagnosed. The present study retrospectively analyzed the clinical data and follow-up results of patients with bladder paraganglioma that were treated surgically in Peking University Shenzhen Hospital between 2012 and 2018, and reviewed the relevant literature. A total of 4 patients with bladder paraganglioma were treated surgically from 2012 to 2018. All patients were female with ages ranging from 28 to 54 years old (average, 47.25). A total of one patient exhibited micturition attacks and palpitations, and one other exhibited gross hematuria. A total of two patients exhibited while no obvious symptoms. One patient exhibited elevated plasma catecholamine, was well prepared with medicine and underwent partial cystectomy, and 3 patients were not successfully diagnosed with paraganglioma before surgery. These patients received transurethral resection and 2 had distinct blood pressure elevation during operation. After surgery, the patients were followed-up for 5-69 months, during which all had normal blood pressure and none experienced recurrence. Bladder paragangliomas exhibit a variety of clinical presentations, which can be nonspecific for the non-functional ones, therefore may easily lead to misdiagnosis. Surgeons must fully consider the possibility of this disease when dealing with non-epithelial bladder tumors. Surgical resection is an effective treatment. For cases successfully diagnosed before surgery, partial cystectomy should be a treatment priority.
\end{abstract}

Correspondence to: Dr Hang Li or Professor Yongqing Lai, Department of Urology, Institute of Urology, Peking University Shenzhen Hospital, Shenzhen PKU-HKUST (Peking University, Hong Kong University of Science and Technology) Medical Center, 1120 Lianhua Road, Shenzhen, Guangdong 518036, P.R. China E-mail: hangtian21@163.com

E-mail: yqlord@163.com

Key words: paraganglioma, bladder tumor, diagnosis, immunohistochemistry, treatment

\section{Introduction}

Paraganglioma, referred to as chromaffin cell-related tumor located outside the adrenal gland (1), originates from the neuroectoderma cells of the sympathetic nervous system (2) and may occur in retroperitoneum, mediastinum, skull and also bladder (3). Accounting for about $10 \%$ of extraadrenal paraganglioma and $0.06 \%$ of all bladder tumors $(1,4)$, bladder paragangliomas are very rare, with only about 200 cases reported worldwide for now (5). They may occur in patients of all ages, but typically at the age of 20-50 years, and more in female patients than male ones (5).

Bladder paragangliomas can be occasionally found during physical examination, presenting with no clinical symptoms, and also may show paroxysmal hypertension, hematuria and other clinical manifestations (6). Because of the low incidence and usually atypical symptoms, they are easily misdiagnosed and may further lead to trouble with treatment. Surgical resection is the most effective way of treatment, but the approaches of surgical resection remain contradictory. Most urologists believe partial cystectomy is the safer way of tumor resection, but there are also many proponents of transurethral resection, claiming it's more minimally invasive and also safe enough (7). Therefore, we summarized and analyzed the 4 cases of bladder paraganglioma surgically treated at this institution in recent years, in order to help urologists with management of similar cases.

\section{Patients and methods}

Clinical data of all the bladder paraganglioma patients who underwent operation in recent years in Peking University Shenzhen Hospital were reviewed. All cases were confirmed pathologically. We summarized the key points of clinical diagnosis and treatments of bladder paraganglioma by analyzing their epidemiological features, symptoms, imaging, laboratory tests, treatments, pathology and immunohistochemistry, and also follow-up outcomes. The informed consent was obtained from every patient, and the study was approved by the Ethics Committees of Peking University Shenzhen Hospital.

\section{Results}

In total, there were 4 bladder paraganglioma patients who underwent surgical treatments from 2012-2018 at this institution. 
All of them were female. Their ages ranged from 28-54 years, averagely 47.25 years old. The following are their case reports.

Case 1. No. 1 patient was a 54 years old woman. The patient complained of paroxysmal headache and palpitation, which often occurred during urinating, together with cold limbs, sweating and hypertension $(220 / 115 \mathrm{mmHg})$, and usually disappeared spontaneously in 2-3 min. Enhanced computed tomography (CT) (Fig. 1A) before operation revealed a solitary, round-like and intensely enhancing lesion located on the posterior wall of bladder, with a size of $25 \times 10 \mathrm{~mm}$. The tumor showed smooth surface in cystoscopy examination. Because of the typical symptoms, she was suspected as paraganglioma and her blood catecholamine level was evidently elevated when tested at headache attacks. Diagnosed successfully before surgery, she was fully prepared by volume hydration, phentolamine and other anti-hypertensive medication. Then she underwent partial cystectomy and the tumor was removed successfully and completely, with her heart rate and blood pressure stable during the operation. Pathological features are shown in Fig. 2A and further immunohistochemical tests revealed that the tumor was positive for chromogranin A (CgA) and synaptophysin (Syn), but CD56 was not did for her. The follow-up lasted for 55 months, during which she had normal blood pressure and no recrudescence was found.

Case 2. No. 2 patient was 53 years old, female. The main symptom was painless grass hematuria, with no urethral irritation symptoms or hypertension. Her CT (Fig. 1B) revealed a $30 \times 20 \mathrm{~mm}$ solitary lesion located on the bladder neck. The tumor had a follicular appearance in cystoscopy. Due to the absence of symptoms, she was not suspected as paraganglioma before surgery. No blood catecholamine test or medical preparation was did for her. Then she underwent transurethral resection, during which she experienced elevated blood pressure and increased heart rate distinctly, with complaints of palpitation and nausea. Nevertheless, the operation was successfully finished with the help of medical control. No complications occurred after surgery. Her pathological features are shown in Fig. 2B and immunohistochemical tests of CgA, Syn and CD56 were positive. She was followed up for 23 months and had no high blood pressure or tumor recrudescence.

Case 3. No. 3 patient was 54 years old and also female. She was identified of bladder tumor on a routine physical examination and had no obvious symptoms or hypertension. Enhanced CT (Fig. 1C) showed a 10x6 mm solitary lesion on the right side wall of bladder and the tumor surface looked smooth in cystoscopy. Diagnosed as common bladder tumor before surgery, no blood catecholamine test or medical preparation was did for her due to the absence of symptoms. She underwent transurethral resection had no elevated blood pressure or other problems during the operation. After surgery, the pathological (Fig. 2C) and further immunohistochemical reports revealed paraganglioma as CgA, Syn and CD56 were positive. The follow-up lasted for 6 months and no hypertension or recrudescence occurred.

Case 4. No. 4 patient was a young female aged 28. She had no obvious symptom or hypertension and her bladder tumor was discovered on a routine physical examination. Further examinations including CT (Fig. 1D) and cystoscopy demonstrated a solitary, round-like and smooth-surface tumor on the right side wall of bladder, with a size of $20 \times 20 \mathrm{~mm}$. She was also not suspected as paraganglioma before surgery and blood catecholamine tests were missed. She underwent transurethral resection as common bladder tumor, and elevated blood pressure and increased heart rate occurred immediately, like no. 2 patient experienced. Luckily the operation was finished with the help of medical control with no complications. The pathological results are shown in Fig. 2D. Immunohistochemical tests revealed that the tumor was positive for $\mathrm{CgA}$, Syn and CD56. We followed up her for 69 months and found no recrudescence or hypertension.

The positive immunohistochemistry for CgA, Syn and CD56 is shown in Fig. 3, but we lack figure evidence for urothelium cytokeratin negativity but only reports.

To make the compares clearer, we summarized their data in Table I.

\section{Discussion}

Paraganglioma is a very rare kind of neoplasm, accounting for $10 \%$ of all chromaffin tumors approximately. It is estimated that there are only about 800 cases diagnosed in the USA every year (8). Paraganglioma is derived from chromaffin tissues of the autonomic nervous system and therefore often occurs along the sympathetic chain or the organ of Zuckerkand (3), widely distributed in many positions like retroperitoneum, bladder, paraaorta, and pelvic cavity (9). Paragangliomas in urinary bladder are even rarer, accounting for $10 \%$ of all paraganglioma and $0.06 \%$ of all bladder tumors $(1,4)$. Since Zimmerman described the first bladder paraganglioma in 1953 (10), only 200 cases have been spotted globally (5). Bladder paragangliomas can be found in all ages, ranging from 11-84 years as reported, but mostly at the age of 20-50 years (5). Females seem to have a higher incidence than male, and the ratio is about 3:1 (11). The 4 cases at this institution were all females, aged 28-54 years. Considering the number is too few, this situation basically correspond to the literature.

Bladder paragangliomas can be functional or non-functional according to whether catecholamine is over-secreted. Most of them are functional (83\%) (12), with main symptoms include persistence or paroxysmal hypertension, headache, palpitation and sweating (13). Paroxysmal hypertension particularly occurs during micturition, which triggers the secretion of catecholamine due to increased bladder pressure caused by bladder contraction, and therefore also called as 'micturition attacks'. Other common symptoms of bladder paragangliomas are painless gross hematuria (55-58\%), lower urinary tract irritation symptoms (about 10\%) (14), and so on. Non-functional bladder paragangliomas can be easily misdiagnosed as bladder cancer before surgery, especially for those with gross hematuria as main complaint. It is reported that only $28.9 \%$ of bladder paraganglioma were successfully diagnosed before surgery, and $61.6 \%$ were initially suspected as bladder cancer or intramucosal bladder tumor (15). No. 1 of our patients had typical symptoms like paroxysmal hypertension and was successfully diagnosed and fully prepared preoperation, while the others had no obvious symptom and were finally 
A

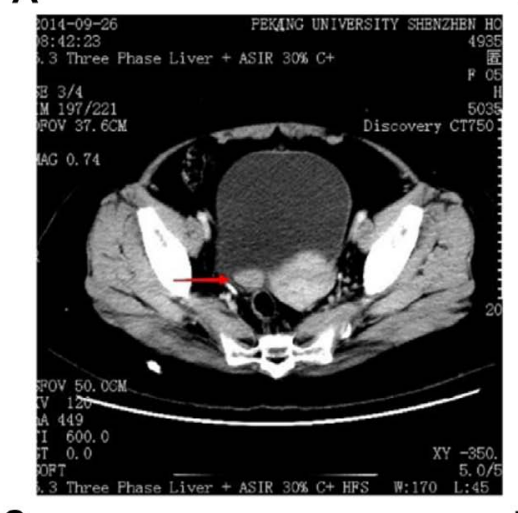

C

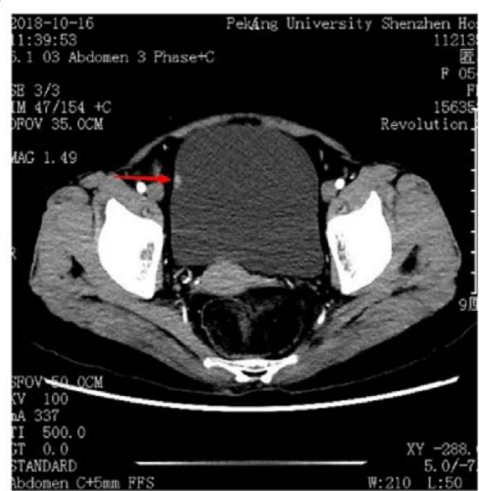

B

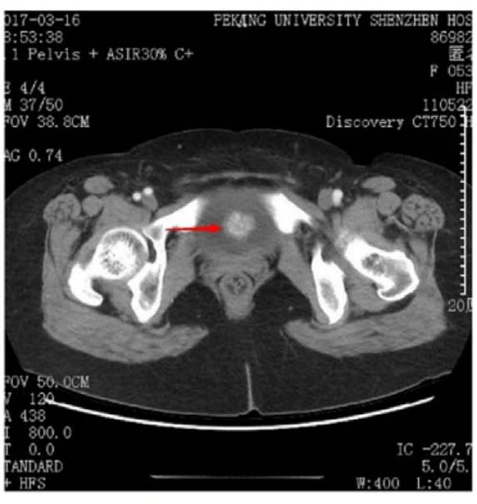

D

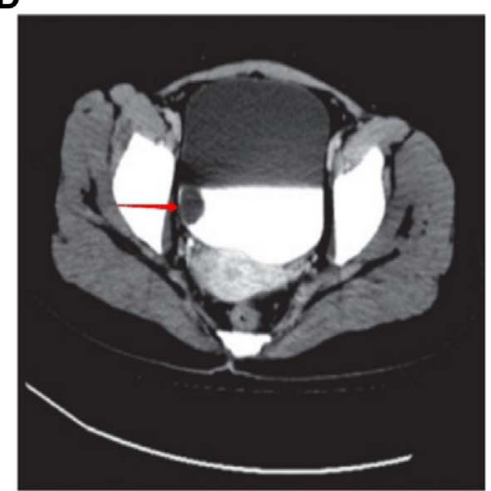

Figure 1. Enhanced CT of bladder paraganglioma (transverse section). Red arrows indicate the location of the tumors. (A) Patient 1, tumor on posterior wall of bladder, 25x10 mm. (B) Patient 2, tumor on bladder neck, 30x20 mm. (C) Patient 3, tumor on right-side wall of bladder, 10x6 mm. (D) Patient 4, tumor on right-side wall of bladder, $20 \times 20 \mathrm{~mm}$ (excretion phase).

\section{A}

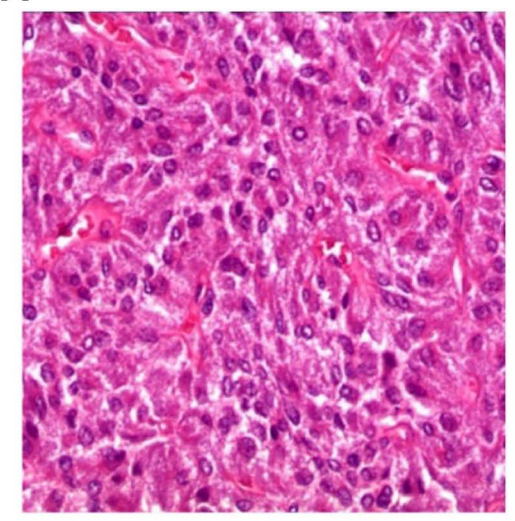

C

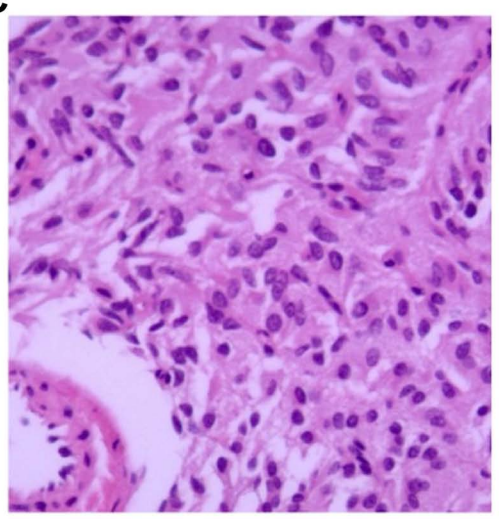

B

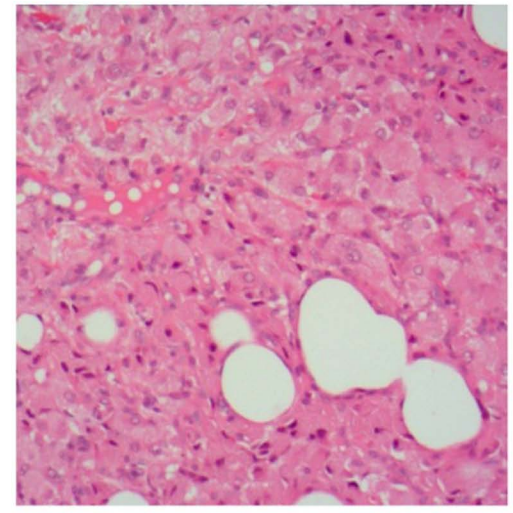

D

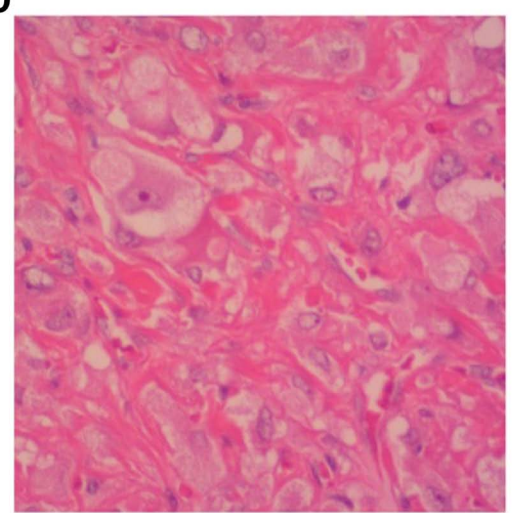

Figure 2. Histopathological evaluation of bladder paraganglioma (H\&E staining; magnification, $\mathrm{x} 400$ ). (A) Patient 1, spindle or round tumor cells with basophilic, granular cytoplasm and round nucleus, abundant vessels. (B) Patient 2, diffuse polygonal tumor cells. (C) Patient 3, beam-like or nest-pattern cells, with small round nucleus and rich cytoplasm. Abundant blood vessels. (D) Patient 4, nest-pattern and polygonal tumor cells. 
A

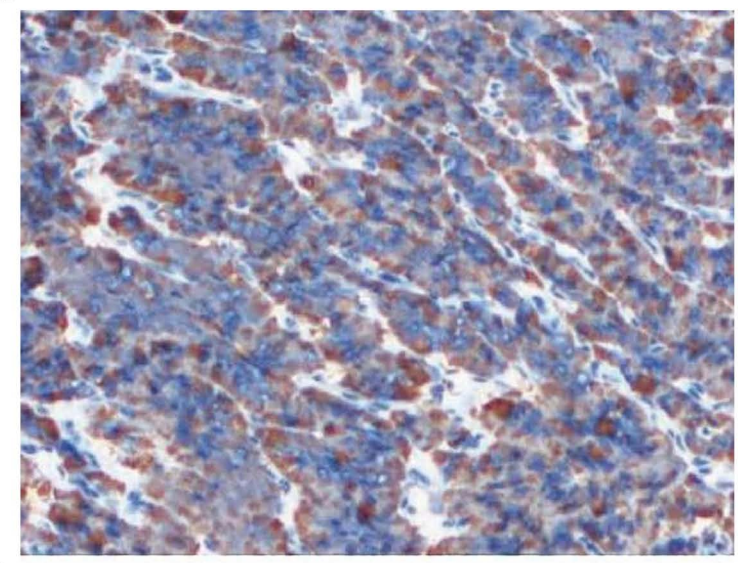

B

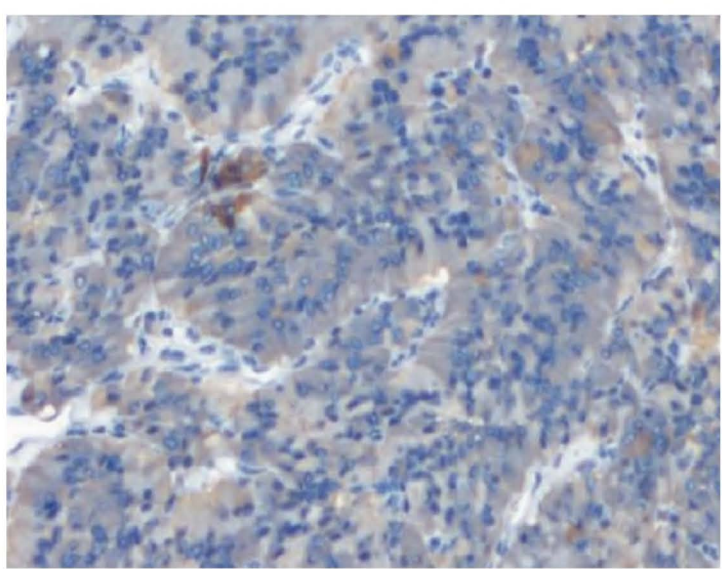

C

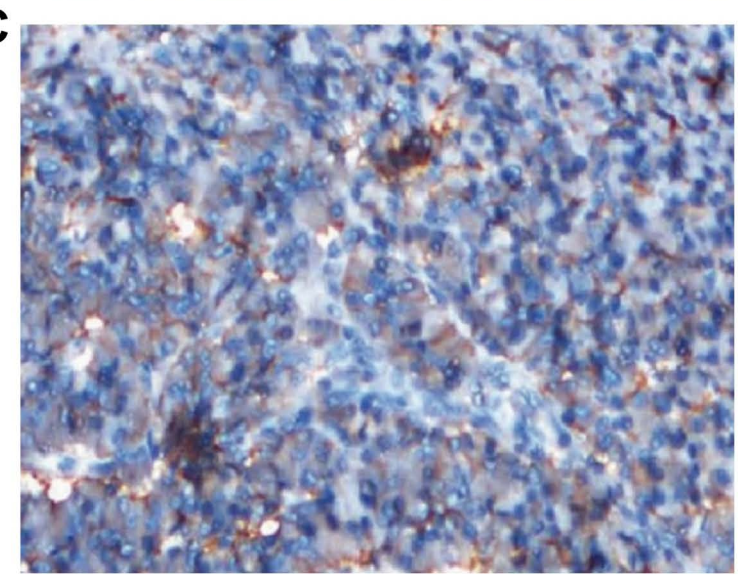

Figure 3. Immunohistochemistry evaluation of bladder paraganglioma (magnification, x200). (A) Positive staining for chromogranin A. (B) Positive staining for synaptophysin. (C) Positive staining for CD56. CD56, cluster of differentiation 56 .

diagnosed by postoperative pathology and immunohistochemistry. This reminds urology surgeons that when finding non-epithelial bladder tumors, paraganglioma must be taken into consideration.

Adequate medical preparation is very important before surgery, or hypertensive crisis may happen during the operation and the mortality risk will increase correspondingly. Therefore, when paraganglioma is suspected, some endocrine tests must be conducted, including methoxyadrenaline, adrenaline, norepinephrine, dopamine and vanillylmandelic acid (VMA) of blood and urine. However, as catecholamine is secreted intermittently, positive rate of direct test is too low. It is reported that only about $60 \%$ of paraganglioma cases can be found of ascended urine VMA and blood catecholamine (15), which makes the differential diagnosis quite difficult. To improve the accuracy, respective blood catecholamine tests before, during and after micturating are suggested (16).

Imaging examinations are also very important for diagnosis. Ultrasound and CT / Magnetic Resonance Imaging (MRI) are the basic estimated methods for the shapes, sizes and locations of the tumors. Ultrasound usually shows clear boundary, wide base and abundant blood supply. On CT scanning, they mostly have equal or slightly higher density (17). The characteristic feature of MRI is slightly higher signal on T1W1, which is just opposite of adrenal pheochromocytoma, and marked enhancement on enhanced scanning (18). For the diagnosis of bladder paraganglioma, CT and MRI have a sensitivity as high as $90-100 \%$, but relatively lower specificity of $70-80 \%$ (19). Besides, some functional imaging technology can also describe anatomy and function situation of the tumors and provides even higher value when judging whether tumors are multiple or metastatic. For example, I131-methyliodobenzylguanidine (131I-MIBG) and positron emission tomography (PET) have a sensitivity of $80-95 \%$ for diagnosis and locating and are very effective for finding possible metastasis (20). But they are used not as extensive as CT or MRI, mainly limited by the equipments and high costs.

Pathologically, the tumor cells usually grow in a characteristic nested pattern, with full and sometimes granular cytoplasm and surrounded by rich blood vessels (21). But they are still easily confused with urothelial carcinoma because many of them also show features like diffuse growth, necrosis and lamina propria infiltration $(22,23)$. Therefore, immunohistochemistry is extremely significant for the definitive diagnosis. Neuroendocrine markers are the most characteristic, like chromogranin A (CgA), synaptophysin (Syn), neuron-specific enolas (NSE) and CD56 (21), while urothelial markers like cytokeratin (CK) are generally negative (24). Except No. 1 case missed the CD56 test, all our 4 cases are positive in $\mathrm{CgA}$, Syn and CD56 and negative in CK, corresponding to the literature reports.

Surgical resection is the most effective way of treatment, including transurethral resection, partial cystectomy and maybe total cystectomy. If the diagnose of paraganglioma is basically certain before surgery, it is generally believed that partial cystectomy is superior to transurethral resection (25). There are two possible reasons for this. Firstly, transurethral resection will damage the tumor body, which may lead to excessive release of catecholamine and increase the risk of hypertensive crisis (26). Secondly, the tumor usually locates in the very deep layer of bladder wall, therefore transurethral resection may carry the problem of complete resection and increase the potential risk of recrudescence (27). Among the 3 transurethral resection cases at this institution, 2 patients experienced severe hypertension during operation. Even though they were both fortunate enough to avoid any complication with the help of active control, the increasing of risks could not be ignored. Transurethral resection may be suitable for those non-functional, small and well-located ones (7).

Surgical resection generally ensures effective treatment in long term. In a summary involving 75 successfully 
Table I. Clinical data of patients with bladder paraganglioma.

\begin{tabular}{|c|c|c|c|c|c|c|c|c|c|}
\hline \multirow[b]{2}{*}{ Number } & \multirow[b]{2}{*}{ Sex } & \multirow[b]{2}{*}{ Age } & \multirow{2}{*}{$\begin{array}{c}\text { First } \\
\text { complacation }\end{array}$} & \multirow{2}{*}{$\begin{array}{l}\text { Tumor } \\
\text { location }\end{array}$} & \multirow{2}{*}{$\begin{array}{c}\text { Tumor } \\
\text { size }(\mathrm{mm})\end{array}$} & \multirow{2}{*}{$\begin{array}{c}\text { Blood } \\
\text { catecholamine }\end{array}$} & \multicolumn{3}{|c|}{ Immunohistochemistry } \\
\hline & & & & & & & $\mathrm{CgA}$ & Syn & CD56 \\
\hline 1 & Female & 54 & $\begin{array}{l}\text { Palpitation, } \\
\text { headache }\end{array}$ & Posterior wall & $25 \times 10$ & Elevated & + & + & None \\
\hline 2 & Female & 53 & Gross hematuria & Bladder neck & $30 \times 20$ & None & + & + & + \\
\hline 3 & Female & 54 & None & Right-side wall & $10 x 6$ & None & + & + & + \\
\hline 4 & Female & 28 & None & Right-side wall & $20 \times 20$ & None & + & + & + \\
\hline
\end{tabular}

CD56, cluster of differentiation 56.

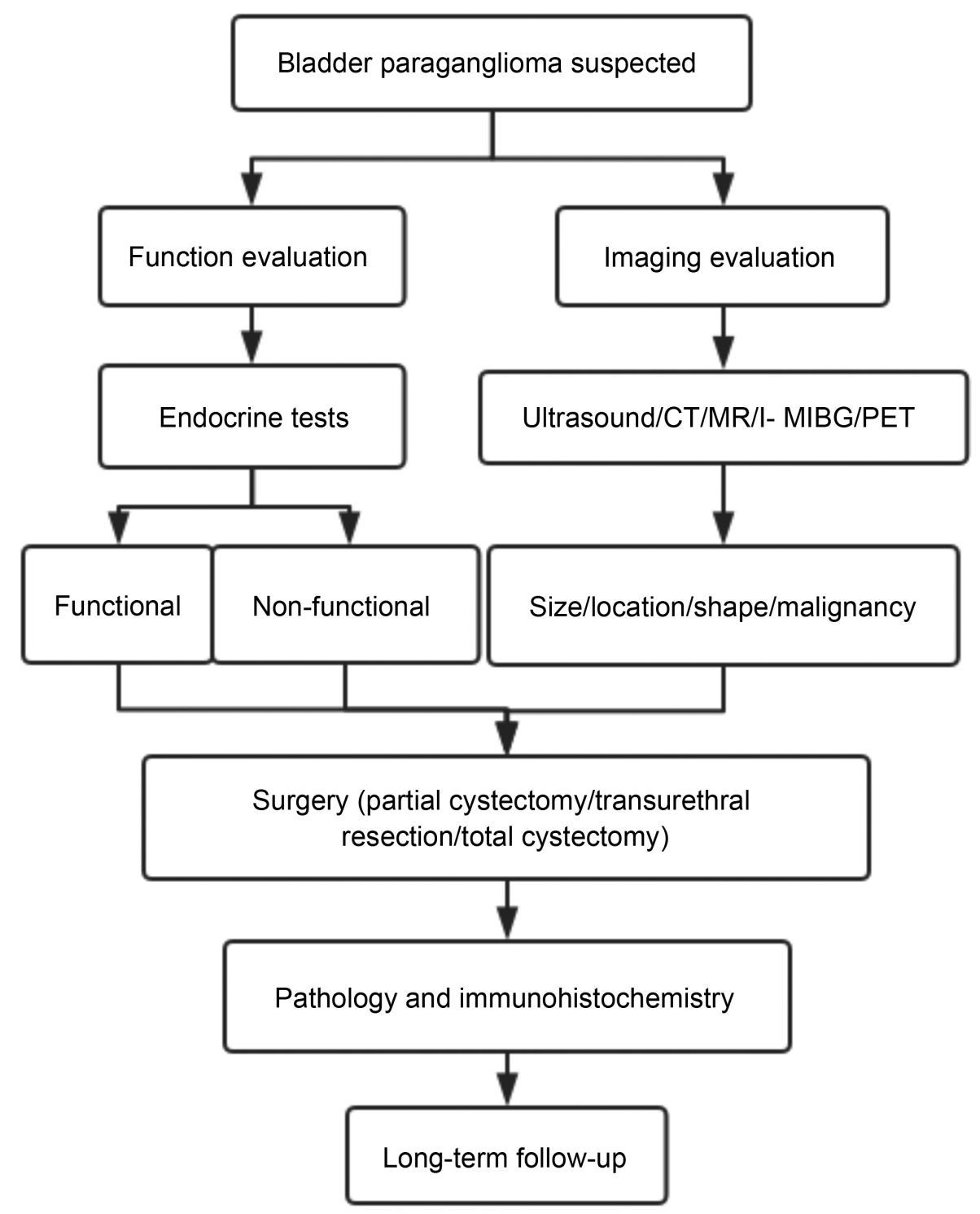

Figure 4. Summary of the diagnosis and treatment steps. When bladder paraganglioma is suspected, function and imaging evaluation will be taken, followed by surgical treatment. Post-operational pathology and immunohistochemistry will determine the diagnosis. Long term follow-up is recommended.

followed-up cases, about $20 \%$ patients experienced disease recurrence or metastasis at the time of last follow-up (5). None of our 4 patients were found of recurrence or metastasis in the 5-69 months of follow-up. Patients with metastatic tumors can hardly get cured by surgery, but they can still benefit form surgery by distracting tumor burden and reducing complications like hypertension. Radiotherapy and chemotherapy are also necessary for metastatic cases and detailed plan should 
be made according to the specific situation of the patient and disease $(28,29)$. Long-term follow-up is recommended for patients with bladder paraganglioma (30). Fig. 4 is a diagram that summarize the diagnosis and treatment steps highlighted in our discussion.

In summary, bladder paraganglioma is a very rare disease with various complications like paroxysmal hypertension, hematuria and some other symptoms, therefore many are easily misdiagnosed due to lack of specificity. This disease must be taken into consideration when non-epithelial bladder tumors are found. Imaging examination including CT and MRI and laboratory tests like catecholamine will help to diagnose, but a definitive diagnose often relies on immunohistochemistry. For treatment, surgical resection is the most effective method, mostly with satisfactory results. Adequate reparation before surgery is the key to a steady and successful operation. For the patients successfully diagnosed before surgery, partial cystectomy should be given priority. Some limitations of our study must be considered. Firstly, we lack the figures of urothelium cytokeratin negativity as evidence. Secondly, the number of cases is low because of the rare prevalence. A multicenter study involving enough cases to provide more reliable proof is needed in the future.

\section{Acknowledgements}

Not applicable.

\section{Funding}

The current study was supported by Research Fund Project of Peking University Shenzhen Hospital (grant no. JCYJ2017001) and Clinical Research Project of Shenzhen Health Commission (grant no. SZFZ2018072).

\section{Availability of data and materials}

The datasets used during the present study are available from the corresponding author on reasonable request.

\section{Authors' contributions}

YL made a substantial contribution to the conception and design of this study and performed manuscript review. JX acquired the clinical data and summarized the diagnosis and treatment procedures. SY and ZC analyzed and interpreted the patient data regarding management of bladder paraganglioma. HL participated in the histological examination, was a major contributor in writing the manuscript, and also analyzed and interpreted the data of the study. All authors read and approved the final manuscript.

\section{Ethics approval and consent to participate}

The informed consent was obtained from every patient, and the study was a approved by the Ethics Committees of Peking University Shenzhen Hospital.

\section{Patient consent for publication}

Not applicable.

\section{Competing interests}

The authors declare that they have no competing interests.

\section{References}

1. Lloyd RV, Osamura RY, Klöppel G and Rosai J, (eds): WHO classification of tumours of endocrine organs. 4th edition vol 10, WHO Press, 2017.

2. Young WJ Jr: Paragangliomas: Clinical overview. Ann N Y Acad Sci 1073: 21-29, 2006.

3. Lee KY, Oh YW, Noh HJ, Lee YJ, Yong HS, Kang EY, Kim KA and Lee NJ: Extraadrenal paragangliomas of the body: Imaging features. AJR Am J Roentgenol 187: 492-504, 2006.

4. Leestma JE and Price EB Jr: Paraganglioma of the urinary bladder. Cancer 28: 1063-1073, 1971.

5. Beilan JA, Lawton A, Hajdenberg J and Rosser CJ: Pheochromocytoma of the urinary bladder: A systematic review of the contemporary literature. BMC Urol 13: 22, 2013.

6. Eisenhofer G, Tischler AS and de Krijger RR: Diagnostic tests and biomarkers for pheochromocytoma and extra-adrenal paraganglioma: From routine laboratory methods to disease stratification. Endocr Pathol 23: 4-14, 2012.

7. Ahn SG, Jang H, Han DS, Lee JU and Yuk SM: Transurethral resection of bladder tumour (TURBT) as an optional treatment method on pheochromocytoma of the urinary bladder. Can Urol Assoc J 7: E130-E134, 2013.

8. Elder EE, Elder G and Larsson C: Pheochromocytoma and functional paraganglioma syndrome: No longer the $10 \%$ tumor. J Surg Oncol 89: 193-201, 2005.

9. Jiang LL, Wu HH, Bu N, Zhang JQ, Zhou LQ and Guo XH: Analysis of clinical characteristics of paraganglioma in 42 patients. Zhonghua Yi Xue Za Zhi 98: 280-283, 2018 (In Chinese).

10. Zimmerman IJ, Biron RE and Macmahon HE: Pheochromocytoma of the urinary bladder. N Engl J Med 249: 25-26, 1953.

11. Yadav R, Das AK and Kumar R: Malignant non-functional paraganglioma of the bladder presenting with azotemia. Int Urol Nephrol 39: 449-451, 2007.

12. Al-Zahrani AA: Recurrent urinary bladder paraganglioma. Adv Urol 2010: 912125, 2010.

13. Chaaya G, Morales J, Castiglioni A, Subhani N and Asmar A: Paraganglioma of the urinary bladder: A rare cause of hypertension and urinary tract infections. Am J Med Sci 355: 191-194, 2018.

14. Beysens M, Lapauw B, Gykiere PJ, Dekeyzer S, Lumen N and Decaestecker K: Minimally invasive approach of bladder paraganglioma: Case report and review of literature. Austin J Clin Case Rep 1: 1034, 2014.

15. Iwamoto G, Kawahara T, Tanabe M, Ninomiya S, Takamoto D, Mochizuki T, Kuroda S, Takeshima T, Izumi K, Hattori Y, et al: Paraganglioma in the bladder: A case report. J Med Case Rep 11: 306, 2017.

16. Lecube A, Peña A, Hernández C and Simó R.: Bladder pheochromocytoma: A variation in the plasma catecholamines during micturition. Med Clin (Barc) 112: 477-478, 1999 (In Spanish).

17. Vyas S, Kalra N, Singh SK, Agarwal MM, Mandal AK and Khandelwal N: Pheochromocytoma of urinary bladder. Indian J Nephrol 21: 198-200, 2011.

18. Wang H, Ye H, Guo A, Wei Z, Zhang X, Zhong Y, Fan Z, Wang $Y$ and Wang D: Bladder paraganglioma in adults: MR appearance in four patients. Eur J Radiol 80: e217-e220, 2011.

19. Zeitlin I, Dessau H, Lorberboym M and Beigel Y: Malignant pheochromocytoma of the urinary bladder: Challenges in diagnosis and management. Isr Med Assoc J 13: 311-313, 2011.

20. Furuta N, Kiyota H, Yoshigoe F, Hasegawa N and Ohishi Y: Diagnosis of pheochromocytoma using (123I)-compared with (131I)-metaiodobenzylguanidine scintigraphy. Int J Urol 6: 119-124, 1999.

21. Chen CH, Boag AH, Beiko DT, Siemens DR, Froese A and Isotalo PA: Composite paraganglioma-ganglioneuroma of the urinary bladder: A rare neoplasm causing hemodynamic crisis at tumour resection. Can Urol Assoc J 3: E45-E48, 2009.

22. Zhou M, Epstein JI and Young RH: Paraganglioma of the urinary bladder: A lesion that may be misdiagnosed as urothelial carcinoma in transurethral resection specimens. Am J Surg Pathol 28: 94-100, 2004. 
23. Menon S, Goyal P, Suryawanshi P, Tongaonkar H, Joshi A, Bakshi G and Desai S: Paraganglioma of the urinary bladder: A clinicopathologic spectrum of a series of 14 cases emphasizing diagnostic dilemmas. Indian J Pathol Microbiol 57: 19-23, 2014.

24. Priyadarshi V and Pal DK: Paraganglioma of urinary bladder. Urol Ann 7: 402-404, 2015.

25. Bishnoi K, Bora GS, Mavuduru RS, Devana SK, Singh SK and Mandal AK: Bladder paraganglioma: Safe and feasible management with robot assisted surgery. J Robot Surg 10: 275-278, 2016

26. El-Tholoth HS, Al RS, Alharbi F, Alshammari W, Alzahrani T and Al Zahrani A: Paraganglioma of urinary bladder managed by laparoscopic partial cystectomy in conjunction with flexible cystoscopy: A case report. J Endourol Case Rep 4: 15-17, 2018.

27. Adraktas D, Caserta $M$ and Tchelepi H: Paraganglioma of the urinary bladder. Ultrasound Q 30: 233-235, 2014.
28. Dahm P and Gschwend JE: Malignant non-urothelial neoplasms of the urinary bladder: A review. Eur Urol 44: 672-681, 2003.

29. Ibuki N, Komura K, Koyama K, Inamoto T, Segawa N, Tanimoto K, Tuji M, Azuma H and Katsuoka Y: A pheochromocytoma of urinary bladder treated with neoadjuvant chemotherapy. Hinyokika Kiyo 55: 765-768, 2009 (In Japanese).

30. Zhai H, Ma X, Nie W, Li H, Peng C, Li X, Zhang Y and Zhang X: Paraganglioma of the urinary bladder: A series of 22 cases in a single center. Clin Genitourin Cancer 15: e765-e771, 2017. 